Original Research Paper

\title{
Numerical Study of Static Instability of Pipe Conveying Incompressible Fluid under Different Boundary Conditions
}

\author{
${ }^{1}$ Dahmane Mouloud, ${ }^{2 *}$ Zahaf Samir, ${ }^{3}$ Sid Ahmed Slimane, ${ }^{4}$ Benkhettab Mohamed and ${ }^{1}$ Boutchicha Djilali \\ ${ }^{1}$ Department of Mechanical Engineering, USTO-MB, Oran, Algeria \\ ${ }^{2}$ Department of Technology, University of Djilali Bounaama-Khamis Meliana, Ain Defla-Algeria \\ ${ }^{3}$ Department of Space Mechanical Research, Satellites Development Centre, Algerian Space Agency, \\ BP 4065, Ibn Rochd, USTO, Oran, POS 50 ILOT T12 Bir El Djir, Oran 31130, Algeria \\ ${ }^{4}$ Department of Mechanical Engineering, Mostaganem University-Abdelhamid Ibn Badis, Algeria
}

\author{
Article history \\ Received: 01-11-2020 \\ Revised: 23-11-2020 \\ Accepted: 26-11-2020 \\ Corresponding Author: \\ Zahaf Samir \\ Department of Technology, \\ University of Djilali \\ Bounaama-Khamis Meliana, \\ Ain Defla-Algeria \\ Email: samir.zahaf@univ-dbkm.dz, \\ zahafsamir1983@gmail.com
}

\begin{abstract}
In this article, the influences of uniform velocity profile, mass ratio, length and Winkler elastic foundation on the static instability of pipe conveying incompressible fluid are investigated. The Euler-Bernoulli beam theory is employed to derive partial differential equation of pipes carrying fluid. The results were carried out using ANSYS Workbench program, where the analysis is based on the numerical solution; using Finite element method to formulate both the pipe structure and fluid flow equations. The numerical approach is based on some research and analytical models. The natural frequencies of the system are attained with respect to different boundary conditions, such as pinned-pinned ends, clamped-pinned ends and clampedclamped ends. The numerical results show satisfactory agreement with the theory of many aspects of the pipe dynamical carrying incompressible fluid were observed numerically such as, the increase in flow velocity, mass ratio and length reduced from the rigidity of the system and consequently the proper modes. Winkler elastic foundation has a stabilizing effect on the system.
\end{abstract}

Keywords: Pipe Carrying Fluid, Natural Frequency, Critical Velocity, Instabilities, Elastic Foundation, ANSYS

\section{Introduction}

The problem of pipes conveying fluid has very important role in various industrial applications. They are used in engineering industries, heating exchangers as nuclear production, pipeline and hydropower systems. Predicting the various values prevents engineers and technologists from approaching or falling into the resonance phenomenon. The first study that took care of this topic was by the researcher (Housener, 1952), where he studied the effect of internal flow on the tube behavior (Housener, 1952). Then it is followed by researcher (Paidoussis, 1998; 2004) who published two books in which he collected his various researches with analytical and experimental results, linear and non-linear motion equations using as well as factors affecting this behavior as flow velocity, mass ratio, length, pressure, extension force, gravity and elastic foundation. The stability analysis was used in these studies to calculate the first natural frequency in terms of fluid velocity. The first frequency decreases gradually with the increase in velocity until disappearance, which leads to the instability that corresponds to the first pattern of static instability. We find some research which has incorporated such concepts and analysis similar to (Doaré and de Langre, 2002) in reference; he presented analytical models by calculating the critical velocities of fluid and through it he determined static instability (buckling) and dynamic instability (flutter). Then he followed his research with another work in 2006 dealing with his topic the role of boundary conditions in the instability of onedimensional systems by utilizing a local wave equation (Doaré and de Langre, 2006). In a different way, (Maalawi and Ziada, 2002) presented a mathematical model for determining the critical flow velocity of a pinned-pinned pipe composed of uniform modules, design parameters include the wall thickness and the length of each module (Maalawi and Ziada, 2002). Chellapilla and Simha (2008), studied the effect of two-parameter foundation on the natural frequencies and critical velocities of a fluid-conveying pipe by 
Galerkin method. All of these data and analyzes gave a great impetus to contemporary researchers to study and expand in this field with the great need of the industrial field for such dynamic behaviors. Ni et al. (2011) analyzed the free vibration problem of pipes conveying fluid with several typical boundary conditions and that calculated the first natural frequencies and critical velocity, using semi-analytical method called Differential Transformation Method (DTM). Shaik et al. (2016) presented a research centered around the study of vibrations and instability of straight welded pipe with clamped-clamped ends conveying fluid, using the analytical method and numerical method. Their study is based on an experimental model. Huo and Wang (2016) based on Euler-Bernoulli beam theory and Hamilton's principle to study the dynamic behavior of a vertical conveying-fluid pipe with fixed-free ends. The resulting equation is discretized via the Galerkin approach in which the Eigen-functions of a clampedfree Euler-Bernoulli beam are utilized (Huo and Wang, 2016). Then, the dynamic responses and stability are discussed with regard to the deploying or retracting fluid velocity and mass ratio. Numerical results reveal that the dynamical behavior of the system is mainly affected by the fluid velocity, instantaneous length, mass ratio and gravity. Very soon (2020), the same study repeated with the adoption of the inclination angle between the pipe length and the gravity direction, which are considered to affect the static and dynamic instabilities of the soft pipe. The stability analyses show that, provided that the inclination angle is not equal to $\pi$, the soft pipe is stable at a low fluid velocity and becomes unstable via flutter (dynamic instability) once the flow velocity is beyond a critical value (Chen et al., 2020). Olunloyo et al. (2017) presents an integral transform analytic solution to the equations governing a fluid-conveying pipeline segment where a gyroscopic effect is taken into consideration. The mathematical model idealizes a segment of the pipeline as an elastic beam conveying an incompressible fluid, the results from this study revealed that a continuity profile exists to connect the subcritical, critical and post-critical vibratory behaviors when the absolute values are plotted for any velocity. A very important article was presented by researcher (Rahmati et al., 2018), entitled: Divergence instability of pipes conveying fluid with uncertain flow velocity. The article deals with investigation of probabilistic stability of pipes carrying fluid with stochastic flow velocity in time domain. As a matter of fact, the study has focused on the randomness effects of fluid speed on stability of pipes conveying fluid. The Euler-Bernoulli beam and plug flow theory was adopted in this study to model pipe structure and internal flow, respectively. The effects of boundary conditions, viscoelastic damping, mass ratio and Winkler elastic foundation on the stability regions were discussed. Like the above methods, the experimental approach remains the most effective and most suitable method for many researchers. We find that this method was used by (Jweeg and Ntayeesh, 2016) for estimating buckling critical velocities from measuring several natural frequencies at relatively small flow rates. The results showed good agreement between the estimated and analytical critical velocities in case of pinned-pinned ends and clamped-pinned ends of pipe conveying fluid. For clamped-clamped pipes, the accurate estimation requires higher flow rates.

In this study, interaction between incompressible fluid and structure pipe is based on a procedure of instantaneous coupling, where they obtained load represented by the pressure stemming from flow velocity and is used as extern solicitation for the wall structure numerical model. Unidirectional approach has been used for the three cases; pinned-pinned ends, clamped-pinned ends and clamped-clamped ends.

\section{Equation of Motion}

The physical model of a pipe conveying fluid on Winkler elastic foundation is shown in Fig. 1. We consider a straight pipe with a length $L$, an internal cross-section area $A$, a mass per unit length $m_{s}$ and a flexural rigidity $E I$. The mass per unit length of a conveying fluid $\mathrm{m}_{\mathrm{f}}$ has an axial velocity $U$ varying with time, referring to a Cartesian coordinate system (Oxyz). The pipe is assumed as an Euler-Bernoulli beam initially aligned with the $x$ axis and lateral displacement, whaere the motions are small $(d x \approx \delta s)$. Figure $2 \mathrm{a}$ shows forces on fluid element while, Fig. $2 \mathrm{~b}$ shows forces and moment of pipe element. Linear pipedynamic equations on an elastic foundation have been derived in the previous work (Paidoussis, 1998; 2004):

$$
\begin{aligned}
& E I \frac{\partial^{4} y}{\partial x^{4}}+m_{f} U^{2} \frac{\partial^{2} y}{\partial x^{2}}+2 m_{f} U \frac{\partial^{2} y}{\partial x \partial t} \\
& +\left(m_{s}+m_{f}\right) \frac{\partial^{2} y}{\partial t^{2}}+K y=0
\end{aligned}
$$

The boundary conditions for pinned-pinned pipe are:

$\left.y\right|_{X=0}=\left.\frac{\partial^{2} y}{\partial x^{2}}\right|_{x=0}=\left.y\right|_{x=L}=\left.\frac{\partial^{2} y}{\partial x^{2}}\right|_{x=L}=0$

The boundary conditions for pinned-pinned pipe are: 


$$
\left.y\right|_{x=0}=\left.\frac{\partial y}{\partial x}\right|_{x=0}=\left.y\right|_{x=L}=\left.\frac{\partial^{2} y}{\partial x^{2}}\right|_{x=L}=0
$$

The boundary conditions for clamped-clamped pipe are:

$$
\left.y\right|_{x=0}=\left.\frac{\partial y}{\partial x}\right|_{x=0}=\left.y\right|_{x=L}=\left.\frac{\partial y}{\partial x}\right|_{x=L}=0
$$

We use the same non-dimensional variables and parameters as in reference (Paidoussis, 1998; 2004):

$$
\begin{aligned}
& T=\left(E I /\left(m_{f}+m_{s}\right)^{1 / 2}\right) t / L^{2}, \beta=m_{f} /\left(m_{f}+m_{s}\right), \\
& u=U L\left(m_{f} / E I\right)^{1 / 2}, k=K L^{2} / E I
\end{aligned}
$$

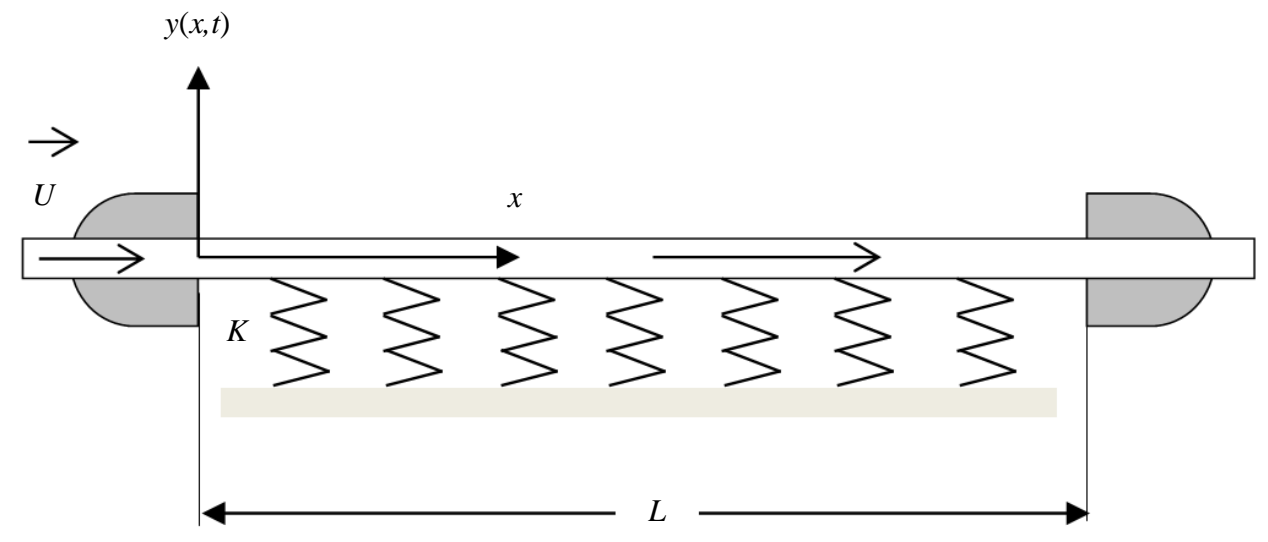

Fig. 1: Representation of the pipe carrying fluid resting on an elastic foundation Winkler-model
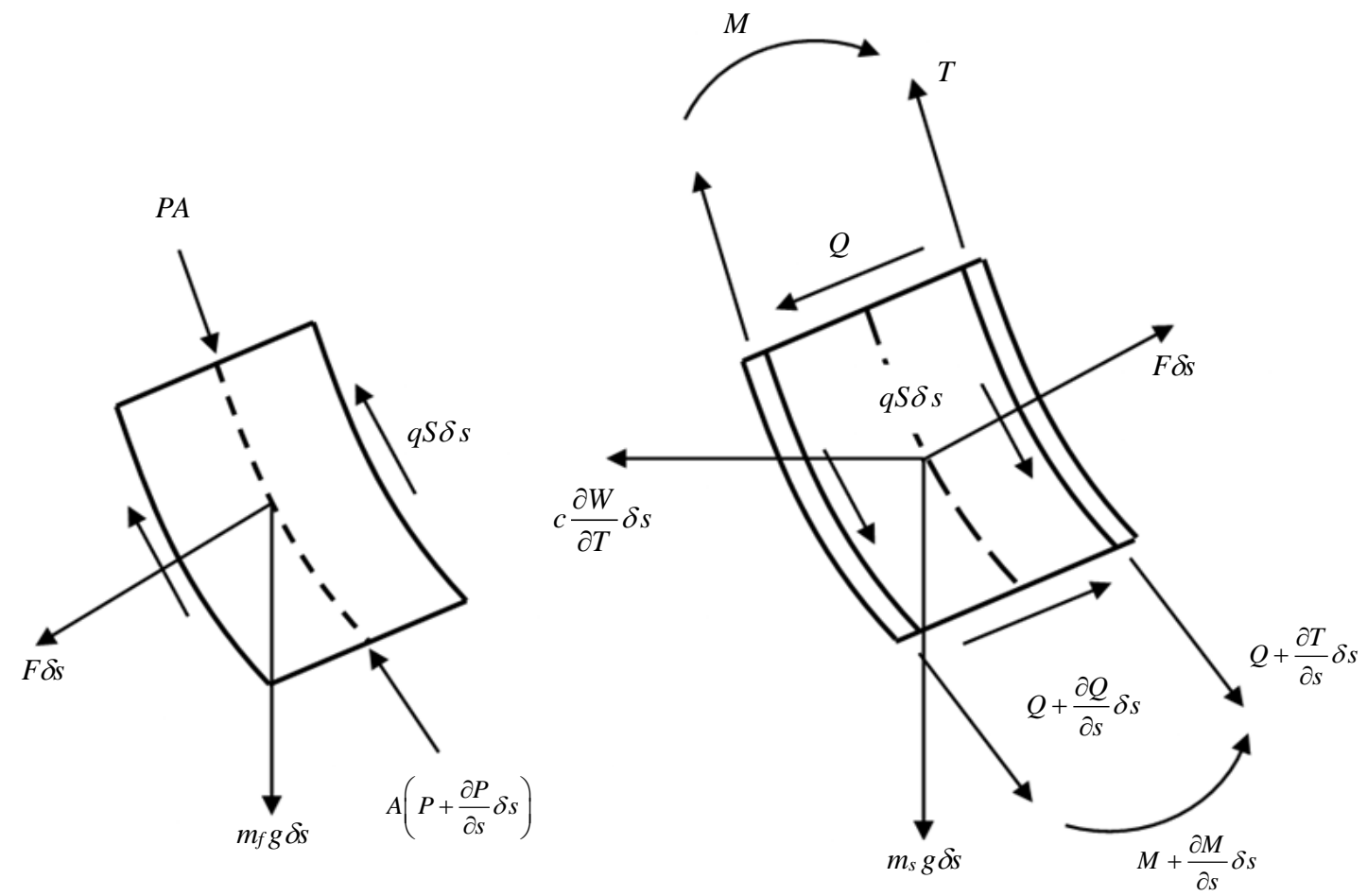

Fig. 2: (a) forces on fluid element; (b) forces and moments on pipe element $\delta_{s}$ (Paidoussis, 2004) 


\section{Method of Resolution}

In this research, we will present the method of calculation and analysis in great details, so that we can know the method of coupling between fluid and pipe structure using the finite element technique and using a widely used program which manifests as ANSYS, Fig. 3. The coupling between the fluid and the structure occurs at the level of the interface between the two domains. The coupling conditions consist of an operation of transfer of the fluid field to the structure and concerns the transfer of pressure stresses to the solid. The Fig. 3 shows a unidirectional simulation. For structure model, we used element with three-dimensional known under SOLID 45, using eight nodes isotropic Fig. 4. The SOLID 45 type enables us to obtain the most natural frequencies of system. For fluid Model, the fluid is incompressible; we used mesh with hexahedral elements Fig. 5.

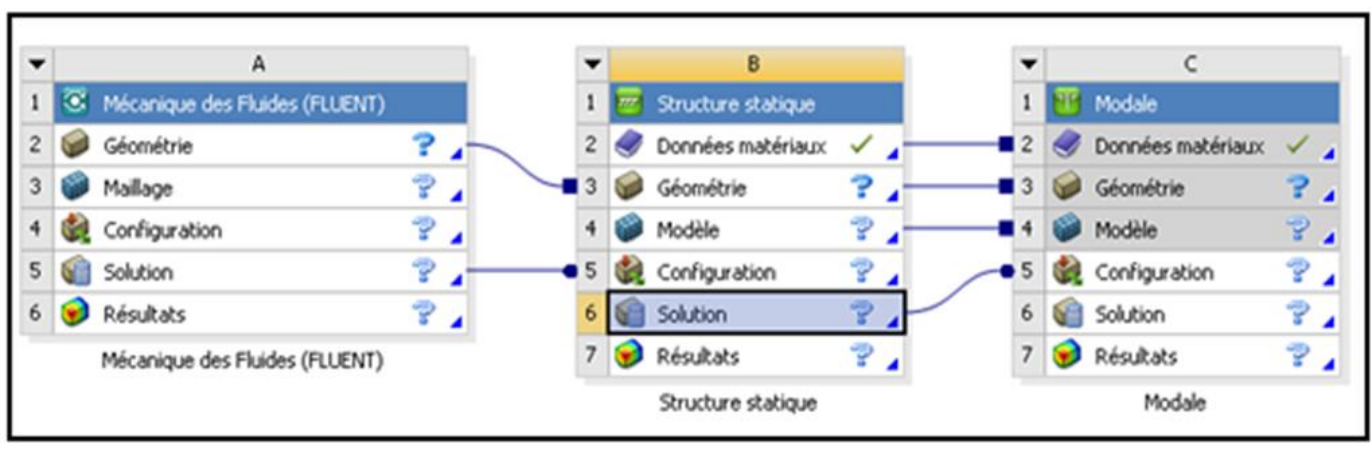

Fig. 3: Unidirectional simulation of fluid-structure interaction

\section{Structure Model}
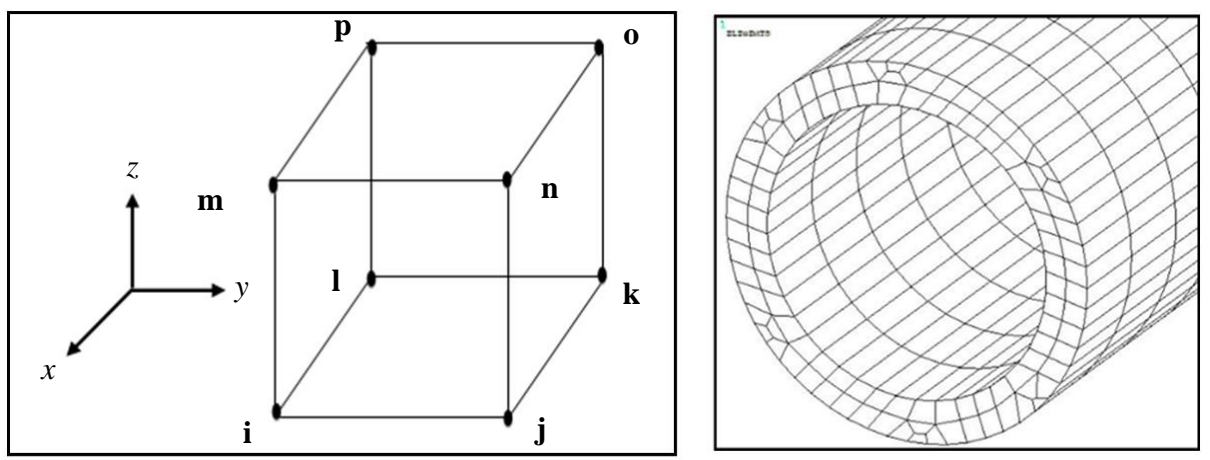

Fig. 4: The profile tube mesh with hexahedral elements (SOLID 45)

\section{Fluid Model}

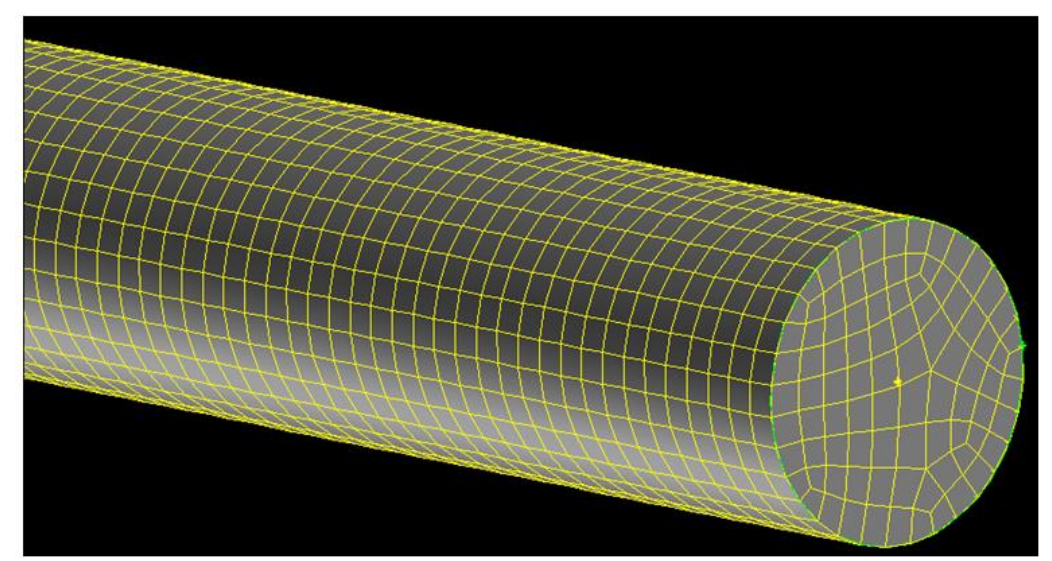

Fig. 5: Volume mesh with 8 nodes-hexahedral 


\section{Entry Condition: Uniform Velocity, $U=10 \mathrm{~m} / \mathrm{s}$}

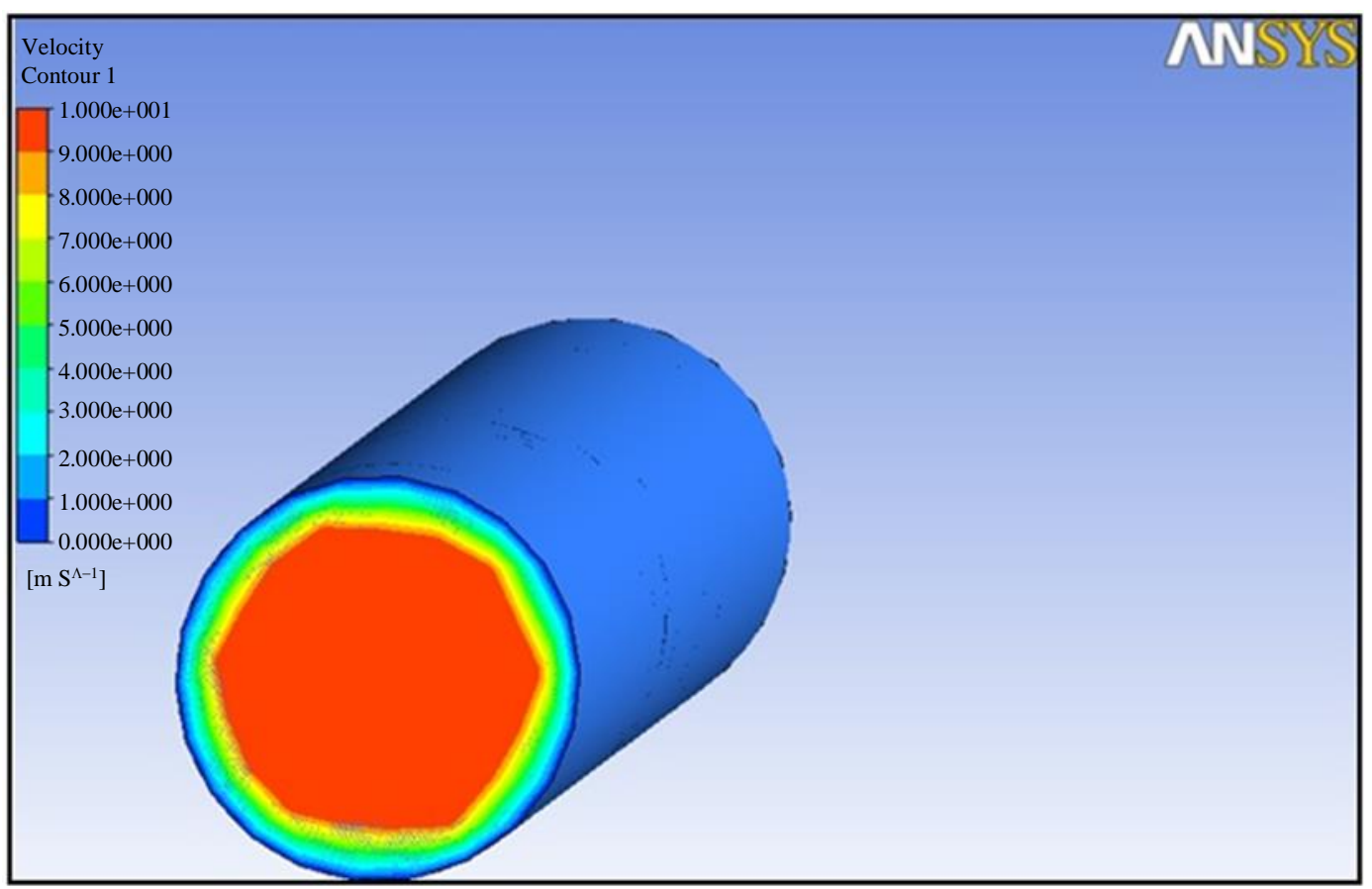

Fig. 6: Iso-values of the input velocity

\section{The State of Convergence}

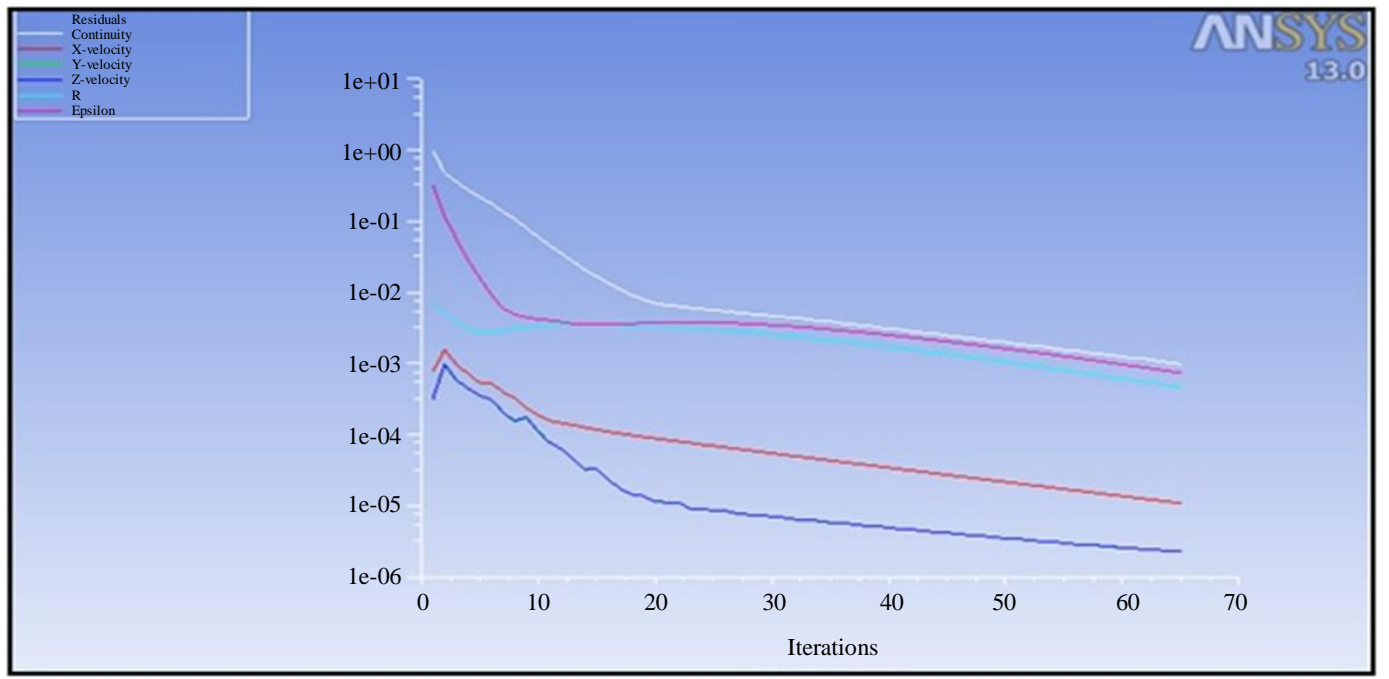

Fig. 7: The state of the residual scale

Figure 6 shows the distribution of the average velocity within the pipe which takes on oval turn, as the velocity distribution is maximum in the middle and less severe beside the wall and this corresponds to the theoretical analysis on the one hand. The Fig. 7 shows the stability of the results (convergence) when this speed is displayed. The flow of fluid gives a pressure, which represents a load distributed along the pipe, Fig. 8. So, the fluid code transmits the pressure, interface node, on pipe structure cod. The structure code calculates the solution from displacement at the nodes as shown in the Fig. 9. The Fig. 10 shows the first natural frequency of clampedclamped pipe with internal flow $(U=10 \mathrm{~m} / \mathrm{s})$, which corresponds to the first mode in bending. 


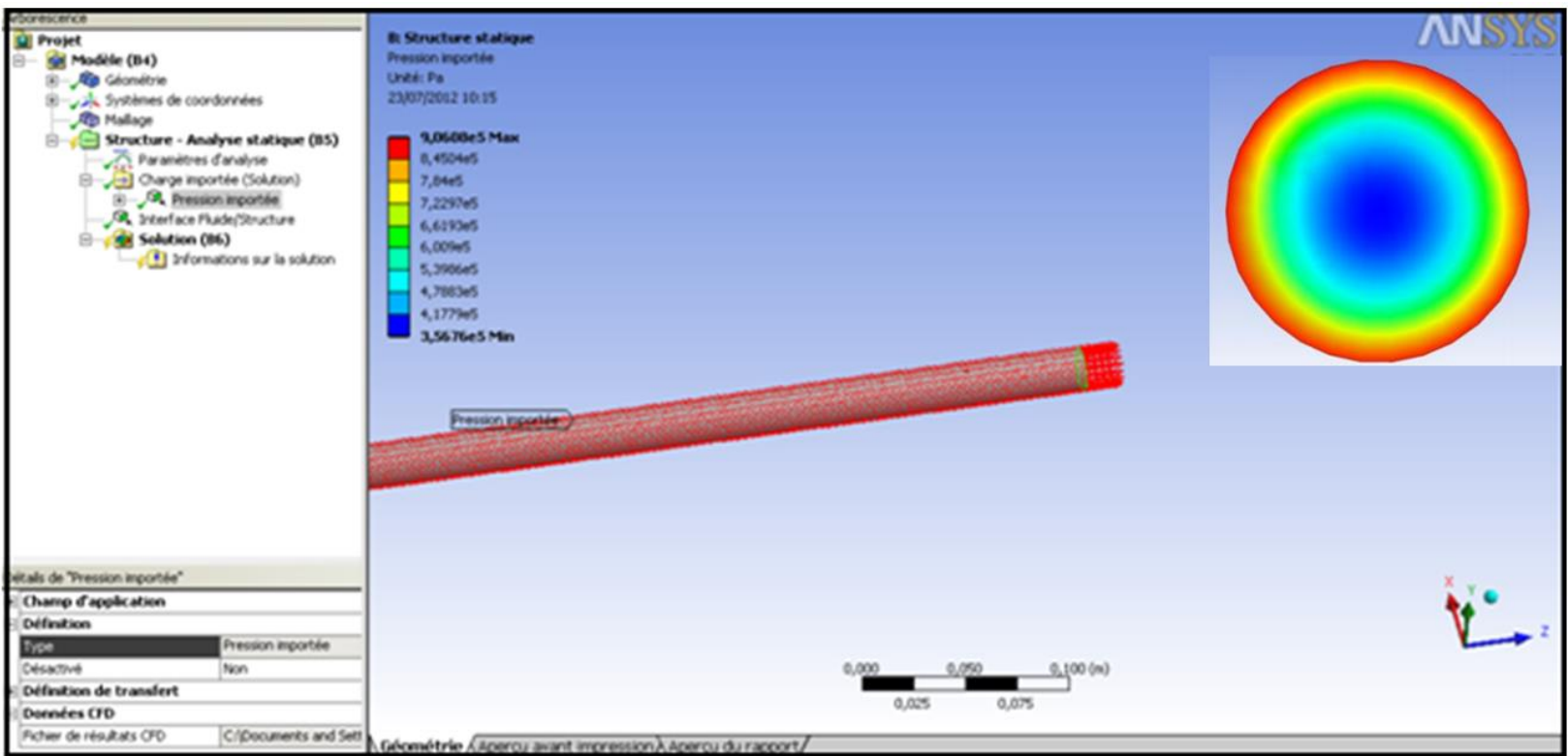

Fig. 8: Imported pressure

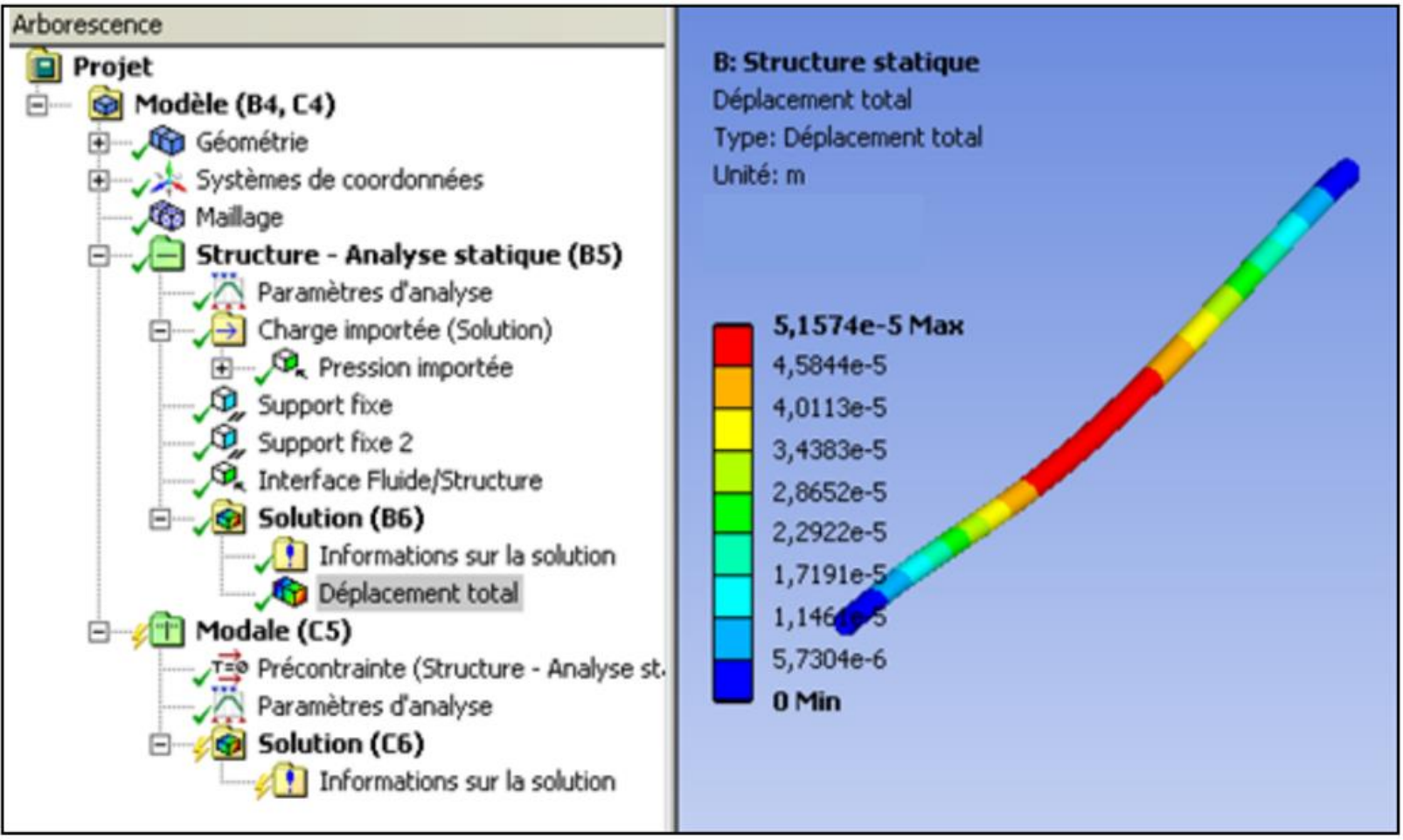

Fig. 9: Iso-values of displacement (mode 1)

The Fig. 11 presents system model within Winkler elastic foundation. The Fig. 12 the first natural frequency of pinned-pinned pipe conveying incompressible fluid on
Winkler elastic foundation, where $(U=10 \mathrm{~m} / \mathrm{s})$. The Fig. 13 Iso-values of the first natural frequency of clamped-pinned pipe with elastic foundation Winkler-model. 


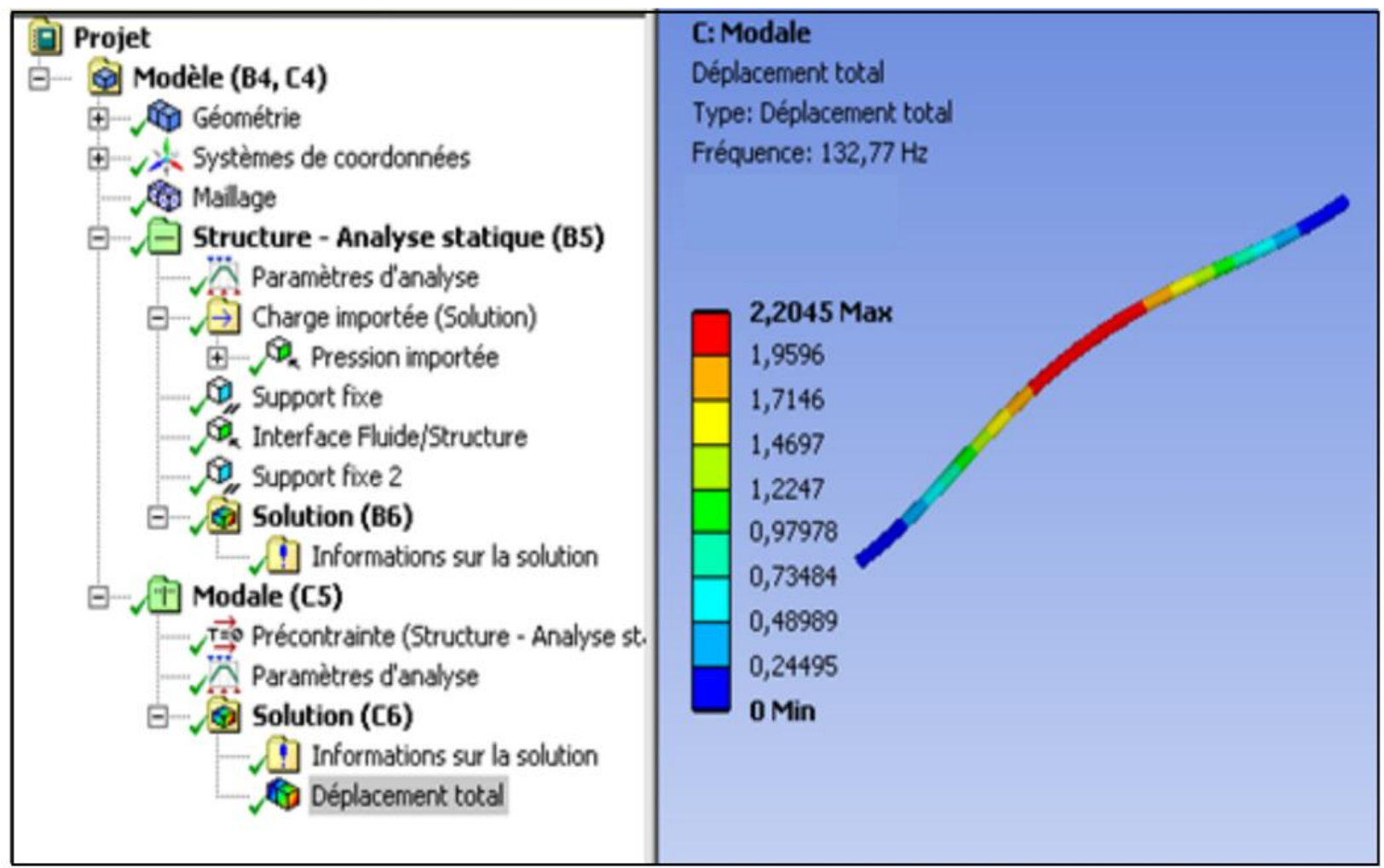

Fig. 10: Iso-values of the first natural frequency of clamped-clamped pipe (mode 1)

Winkler Elastic Foundation

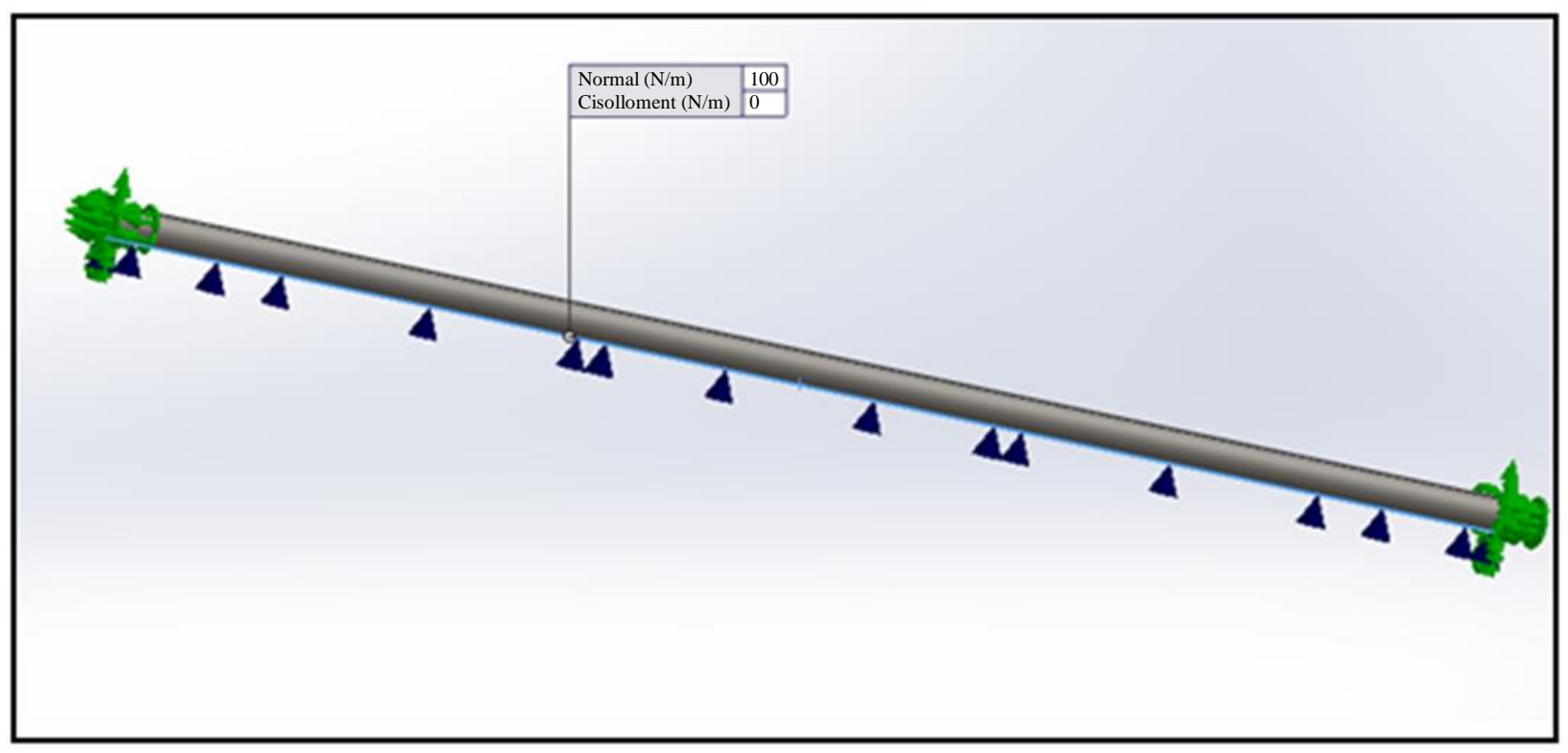

Fig. 11: Representation of the system with an elastic foundation (Winkler-model) 


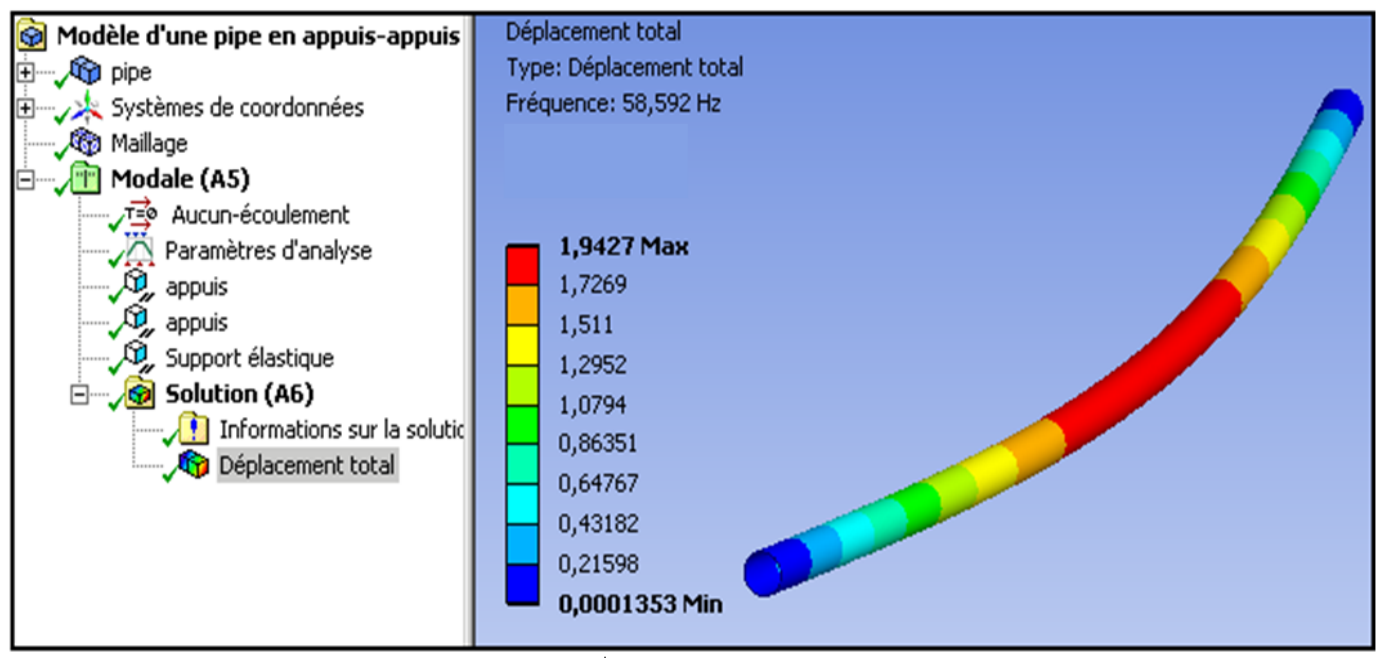

Fig. 12: Iso-values of the first natural frequency of pinned-pinned pipe on elastic foundation (mode 1)

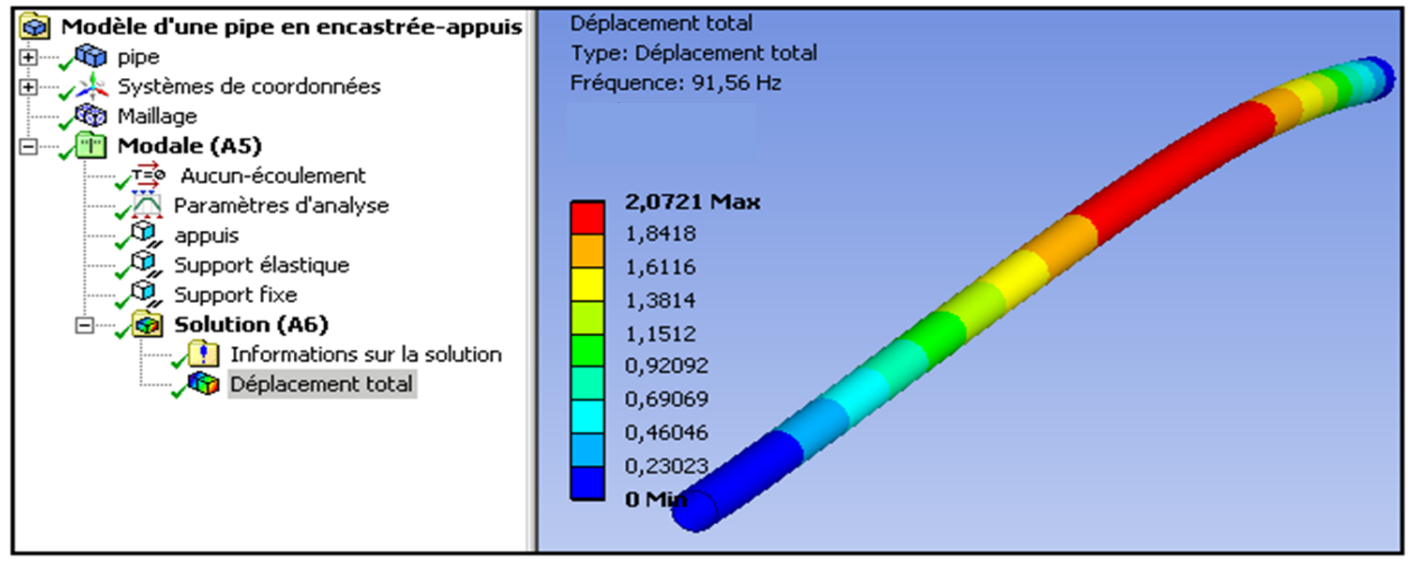

Fig. 13: Iso-values of the first natural frequency of clamped-pinned pipe on Winkler elastic foundation

\section{Discussion}

This research seeks to study the static instability of pipe under internal fluid and this comes by calculating the critical velocity in the static pattern (buckling), that accompanies the disappearance of the first natural frequency in static part. Results will be discussed for various values of flow velocity, mass ratio, length $L$ and elastic foundation for different boundary conditions. The cases can be divided into three according to the parameters effect: Boundary conditions, length and elastic foundation. In the case one and firstly, the numerical results are obtained by DTM and FEM for masse ratio $\beta=0.1$, by using the following boundary conditions: Pinned-pinned ends and on an interval [0 3]. The results obtained are similar, Fig. 14. The same study by experimental approach (Jweeg and Ntayeesh, 2016) and numerical approach (FEM) for $\beta=0.9$, where clamped-pinned ends are presented Fig. 15. Figure 16 shows the first proper mode of clamped-clamped pipe carrying fluid with analytical approach (Rahmati et al., 2018) and numerical method, the results on an interval [0 4] are very close. The results show that the frequency increases with the increase in the rigidity of our system that is evident in the boundary condition type. The system rigidity decreases under the impact of fluid flow, lowering on each boundary pattern. The critical speed of static instability increases with a variation of $57 \%$ between the first two types of boundary conditions and it reaches $31 \%$ between the second and the third. Figures 17-19 present the first natural frequency $(\mathrm{Hz})$ of pinned-pinned pipe under internal flow for different length. Results from the increase in length appear to lead to static instability so, the length has a destabilizing effect on the system. The Fig. 19 shows that the added mass has a significant effect on reducing frequency, as we find that the variation has exceeded $17 \%$ (case: No-flow). The third case deals with the study of the elastic foundation effect on system instability. The Fig. 20 and 21 show the first 
natural frequencies of pipe carrying fluid on Winkler elastic foundation with low velocity $(u=0.1)$, for case: Pinned-pinned ends and clamped-pinned ends, respectively. Table 1 presents the critical velocities for static instability of the pinned-pinned pipe as functions of the foundation stiffness $k$, with $\beta=0.5$. The variation in results is evident, especially for high Winkler parameter. Elastic foundation type of Winkler increases the natural frequencies of the system and consequently the critical velocity.

\section{Numerical Results}

The results are presented in figures from Figs. 14 to 21; they illustrate the frequency developments in terms of several variables in the transmission pipelines using the finite element technique. While Table 1 show the critical velocities of the pinned-pinned pipe carrying flow as functions of the foundation elastic.

Table 1: The critical velocity parameter for various values of Winkler-type

\begin{tabular}{llc}
\hline $\mathrm{k}$ values & Critical velocity & Variation $\%$ \\
\hline 0.1 & 3.142 & 0.12 \\
1 & 3.158 & 1.60 \\
10 & 3.298 & 4.43 \\
100 & 4.472 & 35.59 \\
200 & 5.489 & 22.74 \\
300 & 6.348 & 15.64 \\
400 & 7.049 & 11.04 \\
500 & 7.226 & 2.51 \\
1000 & 8.0596 & 11.53 \\
\hline
\end{tabular}

\section{Boundary Conditions Effect}

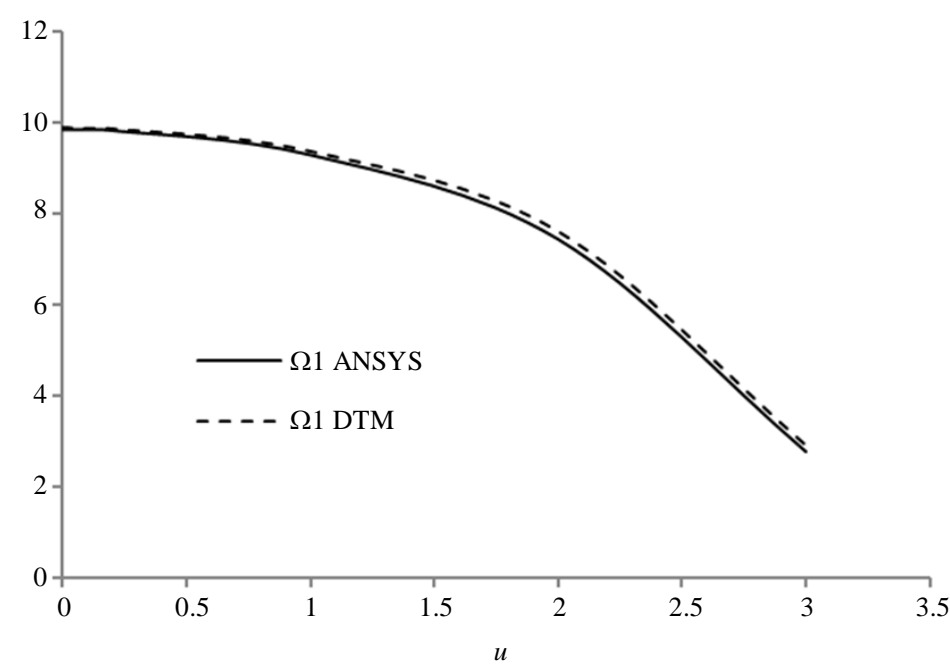

Fig. 14: Natural frequency of pinned-pinned pipe carrying fluid, $\beta=0.1$

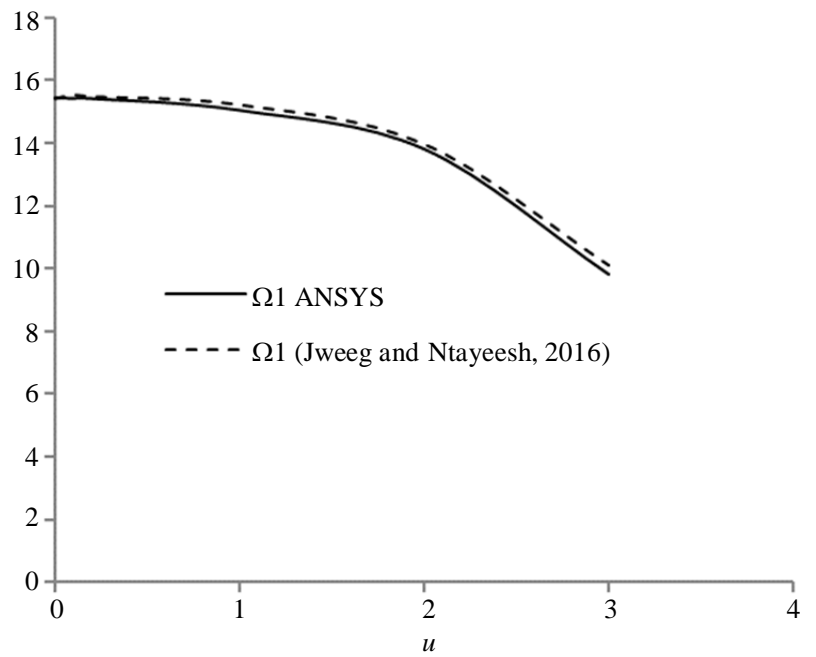

Fig. 15: Natural frequency of clamped-pinned pipe carrying fluid, $\beta=0.9$ 


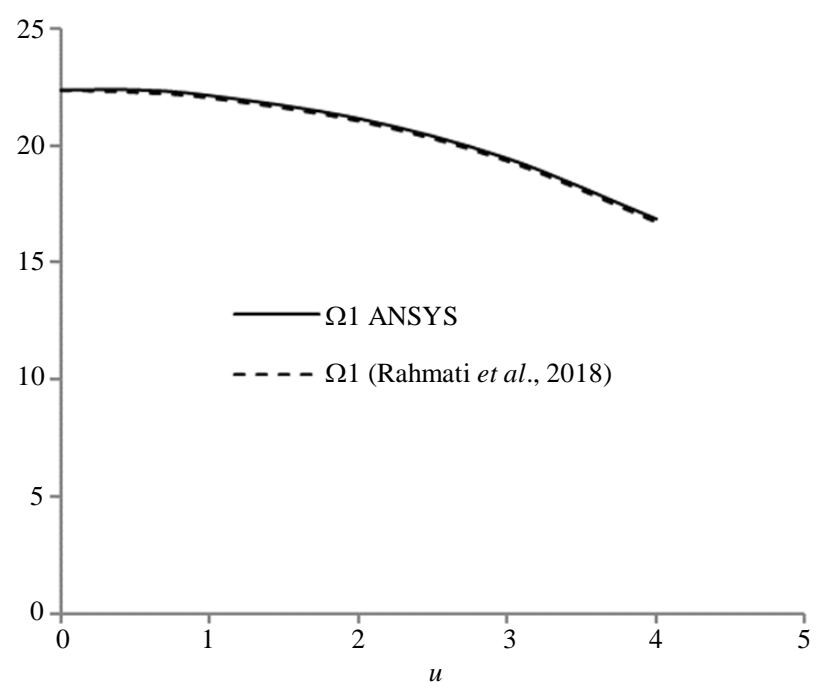

Fig. 16: Natural frequency of clamped-clamped pipe carrying fluid, $\beta=0.3$

\section{Length Effect}

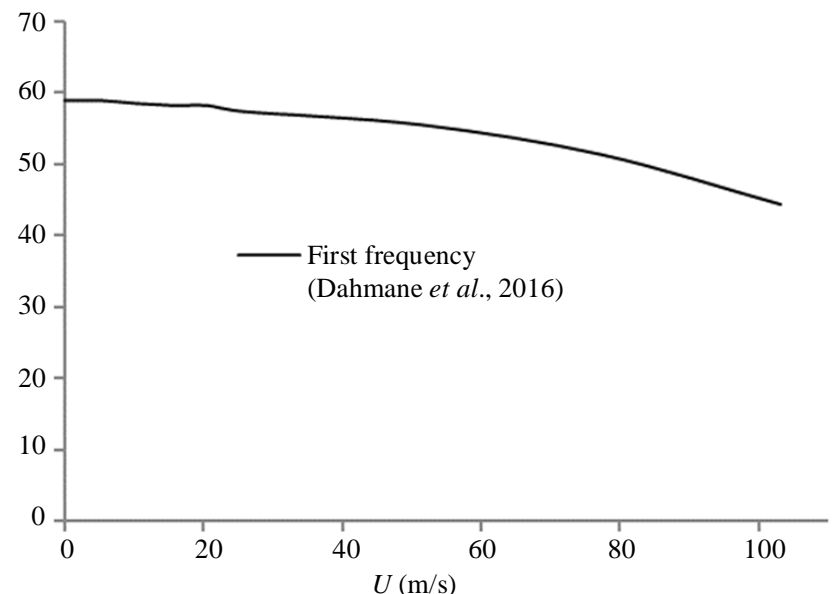

Fig. 17: Natural frequency (Hz) of pinned-pinned pipe carrying fluid, $L=1 \mathrm{~m}$ and $\beta=0.6$

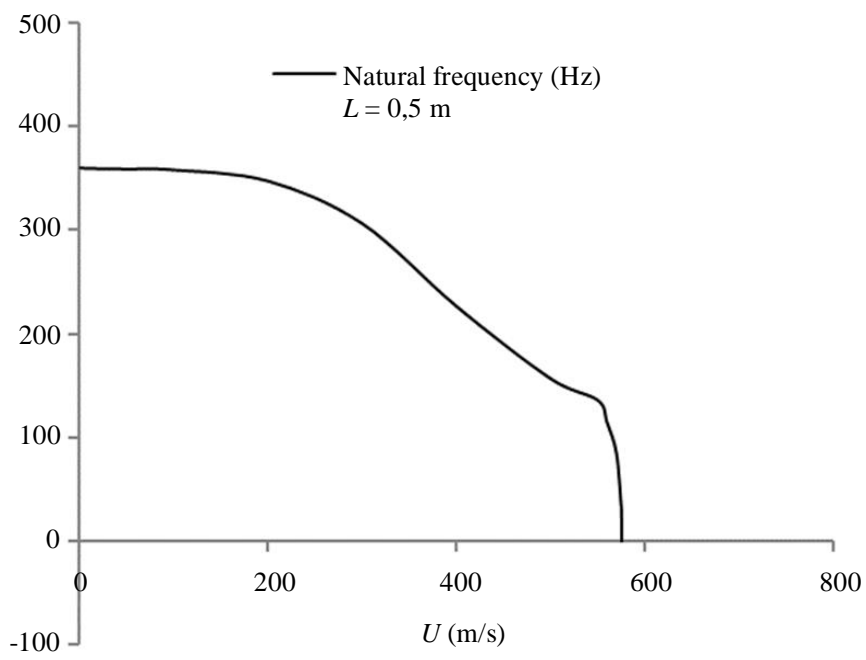

Fig. 18: Natural frequency $(\mathrm{Hz})$ of pinned-pinned pipe carrying fluid, $L=0.5 \mathrm{~m}$ and $\beta=0.6$ 


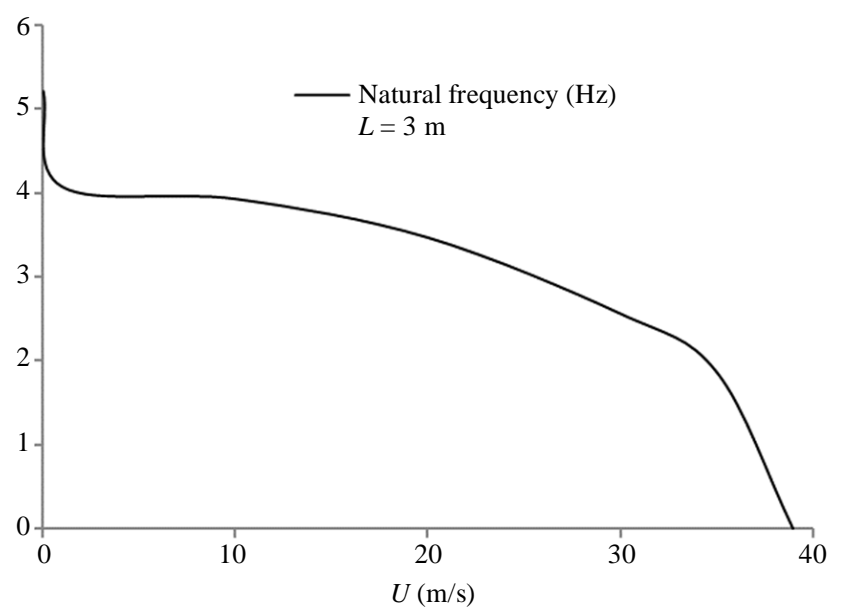

Fig. 19: Natural frequency (Hz) of pinned-pinned pipe carrying fluid, $L=3 \mathrm{~m}$ and $\beta=0.6$

\section{Elastic Foundation Effect}

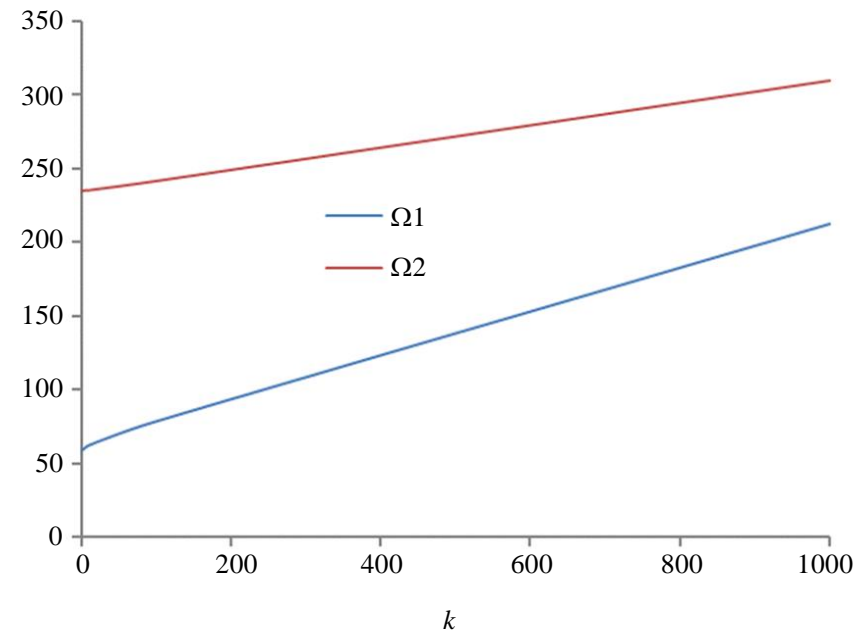

Fig. 20: Natural frequencies of pinned-pinned pipe carrying fluid on Winkler foundation, $L=1 \mathrm{~m}$ and $\beta=0.6$

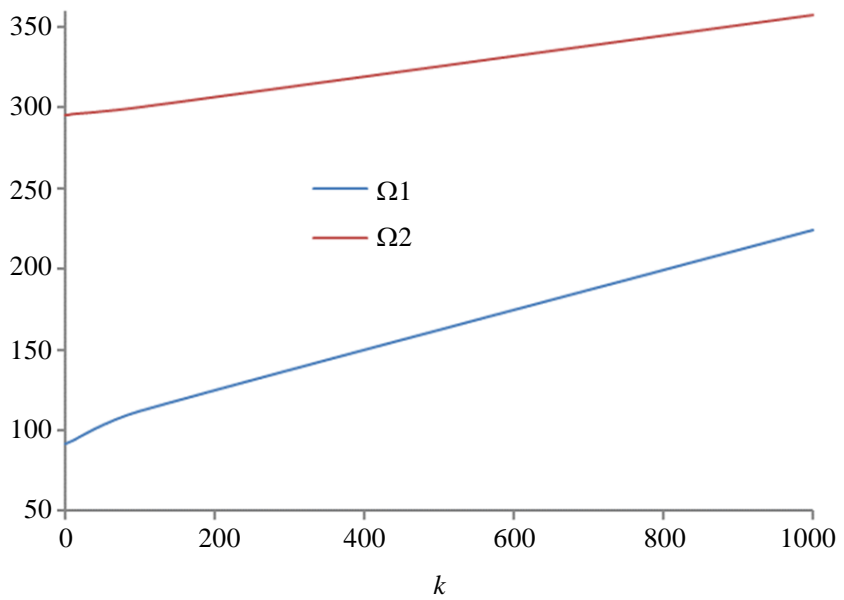

Fig. 21: Natural frequencies of clamped-pinned pipe carrying fluid on Winkler foundation, $L=1 \mathrm{~m}$ and $\beta=0.6$ 


\section{Conclusion}

The present study is a contribution to the study of pipe vibration, hydrodynamic structures, through numerical approach. A tool is used to intend the calculation of the first natural frequencies and visualize the fluid in the pipe system. Numerical results give the natural frequencies and critical velocity that characterized instabilities. We observe that instability appears when the velocity exceeds a threshold called critical velocity of instability, when the first proper mode is zero. Several examples were processed to determine the influence of the fluid velocity and different geometrical and physical parameters on static instability of our system. The boundary conditions play an important role in raising or lowering the critical velocity of fluid that corresponds to the appearance of instability. The length parameter decreases the frequencies and critical fluid velocities and leads to an increase in the static instability. Elastic foundation model of Winkler increases the natural frequencies of the system and consequently the critical velocities.

\section{Author's Contributions}

All authors equally contributed in this study.

\section{Ethics}

This article is original and contains unpublished material. The corresponding author confirms that all of the other authors have read and approved the manuscript and no ethical issues involved.

\section{References}

Chellapilla, K. R., \& Simha, H. S. (2008). Vibrations of fluid-conveying pipes resting on two-parameter foundation. The Open Acoustics Journal, 1(1).

Chen, W., Hu, Z., Dai, H., \& Wang, L. (2020). Extremely large-amplitude oscillation of soft pipes conveying fluid under gravity. Applied Mathematics and Mechanics, 41(9), 1381-1400.

Dahmane, M., Boutchicha, D., \& Adjlout, L. (2016). One-way fluid structure interaction of pipe under flow with different boundary conditions. Mechanics, 22(6), 495-503.
Doaré, O., \& de Langre, E. (2002). Local and global instability of fluid-conveying pipes on elastic foundations. Journal of fluids and structures, 16(1), 1-14.

Doaré, O., \& de Langre, E. (2006). The role of boundary conditions in the instability of one-dimensional systems. European Journal of Mechanics-B/Fluids, 25(6), 948-959.

Housener, G. W. (1952). Bending vibration of a pipeline containing flowing fluid. Trans. ASME, J. Appl. Mech., 19, 205.

Huo, Y., \& Wang, Z. (2016). Dynamic analysis of a vertically deploying/retracting cantilevered pipe conveying fluid. Journal of Sound and Vibration, 360, 224-238.

Jweeg, M. J., \& Ntayeesh, T. J. (2016). Determination of critical buckling velocities of pipes conveying fluid rested on different supports conditions. International Journal of Computer Applications, 134(10), 34-42.

Maalawi, K. Y., \& Ziada, M. A. (2002). On the static instability of flexible pipes conveying fluid. Journal of fluids and structures, 16(5), 685-690.

Ni, Q., Zhang, Z. L., \& Wang, L. (2011). Application of the differential transformation method to vibration analysis of pipes conveying fluid. Applied Mathematics and Computation, 217(16), 7028-7038.

Olunloyo, V. O., Osheku, C. A., \& Olayiwola, P. S. (2017). A Note on an Analytic Solution for an Incompressible Fluid-Conveying Pipeline System. Advances in Acoustics and Vibration, 2017.

Paidoussis, M. P. (1998). Fluid-structure interactions: Slender structures and axial flow (Vol. 1). Academic press.

Paidoussis, M. P. (2004). Fluid-structure interactions slender structures and axial flow, Department of Mechanical Engineering. Academic Press, Elsevier.

Rahmati, M., Mirdamadi, H. R., \& Goli, S. (2018). Divergence instability of pipes conveying fluid with uncertain flow velocity. Physica A: Statistical Mechanics and Its Applications, 491, 650-665.

Shaik, I., Krishnaiah, A., \& Sutar, S. (2016). A Theoretical Study of Flow Induced Piping Vibration with Clamped Ends. Int. J. Mech. Eng. Autom, 3(6), 231-238. 